\title{
Recurrent Neural Network-Predictions for PSO in Dynamic Optimization
}

\author{
Supplementary Material
}

\author{
Almuth Meier \\ University of Oldenburg, Oldenburg, Germany \\ Department of Computing Science \\ almuth.meier@uni-oldenburg.de
}

\author{
Oliver Kramer \\ University of Oldenburg, Oldenburg, Germany \\ Department of Computing Science \\ oliver.kramer@uni-oldenburg.de
}

\begin{abstract}
In this supplementary material, we provide the complete result tables for the analysis of different parameter settings. The tables on the following pages contain the experimental results obtained by executing all PSO variants with different parameter settings as described in the paper.
\end{abstract}

\section{ACM Reference Format:}

Almuth Meier and Oliver Kramer. 2018. Recurrent Neural Network-Predictions for PSO in Dynamic Optimization: Supplementary Material. In GECCO '18: Genetic and Evolutionary Computation Conference, Fuly 15-19, 2018, Kyoto, Japan. ACM, New York, NY, USA, 7 pages. https://doi.org/10. $1145 / 3205455.3205527$
Permission to make digital or hard copies of all or part of this work for personal or classroom use is granted without fee provided that copies are not made or distributed for profit or commercial advantage and that copies bear this notice and the full citation on the first page. Copyrights for components of this work owned by others than ACM must be honored. Abstracting with credit is permitted. To copy otherwise, or republish, to post on servers or to redistribute to lists, requires prior specific permission and/or a fee. Request permissions from permissions@acm.org.

GECCO '18, Fuly 15-19, 2018, Kyoto, Japan

(C) 2018 Association for Computing Machinery.

ACM ISBN 978-1-4503-5618-3/18/07 ..\$15.00

https://doi.org/10.1145/3205455.3205527 
Table 1: Results for dynPSO on SRR benchmark

\begin{tabular}{|c|c|c|c|c|c|c|c|c|c|}
\hline \multirow{2}{*}{\begin{tabular}{|l|} 
Metric \\
Benchmark \\
\end{tabular}} & \multicolumn{3}{|c|}{ BOG } & \multicolumn{3}{|c|}{ BEBC } & \multicolumn{3}{|c|}{ RCS } \\
\hline & 5 & 49 & 0 & 5 & 9 & 0 & 5 & 9 & 0 \\
\hline sph. & $9.2 \mathrm{E}-1$ & $6.0 \mathrm{E}-2$ & $5.4 \mathrm{E}-2$ & 5 & 0.2 & $5.8 \mathrm{E}-5$ & 1 & 1 & 0.03 \\
\hline 5 lin. & $8.2 \mathrm{E}+0$ & $3.1 \mathrm{E}+0$ & $4.3 \mathrm{E}+0$ & $1.9 \mathrm{E}-2$ & $2.7 \mathrm{E}-2$ & $7.2 \mathrm{E}-2$ & 0.02 & 0.02 & .04 \\
\hline ph. 10 lin. & $E+1$ & 2.2E+1 & $9 \mathrm{E}+1$ & 4.1E-1 & $1.4 \mathrm{E}+0$ & $4.1 \mathrm{E}+0$ & .05 & .07 & .12 \\
\hline ph. 20 lin. & $\mathrm{E}+2$ & $1.6 \mathrm{E}+2$ & $3.0 \mathrm{E}+2$ & 2.3E+1 & $3.5 \mathrm{E}+1$ & $9.3 \mathrm{E}+1$ & .15 & .20 & .32 \\
\hline sph. 50 lin. & $6.7 \mathrm{E}+4$ & $3.5 \mathrm{E}+3$ & $6.4 \mathrm{E}+3$ & $6.5 \mathrm{E}+4$ & $2.3 \mathrm{E}+3$ & $4.5 \mathrm{E}+3$ & 89 & .56 & .64 \\
\hline sph. 100 lin. & $3.2 \mathrm{E}+6$ & $.0 \mathrm{E}+4$ & $6.3 \mathrm{E}+4$ & $3.2 \mathrm{E}+6$ & $6.3 \mathrm{E}+4$ & $5.4 \mathrm{E}+4$ & 97 & 33 & .81 \\
\hline sph. & $8.1 \mathrm{E}+0$ & $6 \mathrm{E}-1$ & $3 E-1$ & $1.3 \mathrm{E}-4$ & $1.0 \mathrm{E}-4$ & $1.4 \mathrm{E}-4$ & 01 & 02 & .02 \\
\hline $5 \sin$. & $1.2 \mathrm{E}+1$ & $.7 \mathrm{E}+0$ & $7.2 \mathrm{E}+0$ & $1.9 \mathrm{E}-1$ & $3.6 \mathrm{E}-2$ & $1.8 \mathrm{E}-1$ & .03 & .03 & .05 \\
\hline sph. $10 \mathrm{sin}$. & $4.5 \mathrm{E}+2$ & $9.4 \mathrm{E}+1$ & $1.7 \mathrm{E}+2$ & $2.9 \mathrm{E}+2$ & $5.7 \mathrm{E}+0$ & $1.8 \mathrm{E}+1$ & 15 & .06 & .10 \\
\hline sph. 20 sin. & $1.3 \mathrm{E}+3$ & $4.6 \mathrm{E}+2$ & $8.3 \mathrm{E}+2$ & $9.3 \mathrm{E}+2$ & $1.0 \mathrm{E}+2$ & $2.5 \mathrm{E}+2$ & 31 & 8 & .27 \\
\hline sph. $\quad 50$ sin. & $6.6 \mathrm{E}+4$ & $9.2 \mathrm{E}+3$ & $1.4 \mathrm{E}+4$ & $6.3 \mathrm{E}+4$ & $5.4 \mathrm{E}+3$ & $9.1 \mathrm{E}+3$ & 82 & 7 & .55 \\
\hline sph. $100 \mathrm{sin}$. & $4.9 \mathrm{E}+5$ & 4.1E+4 & $5.2 \mathrm{E}+4$ & $4.8 \mathrm{E}+5$ & $3.3 \mathrm{E}+4$ & $4.2 \mathrm{E}+4$ & 4 & & 0.73 \\
\hline ros. & $3.8 \mathrm{E}+2$ & $3.8 \mathrm{E}+0$ & $1.7 \mathrm{E}+0$ & $4 \mathrm{E}-3$ & $1.2 \mathrm{E}-3$ & $1.5 \mathrm{E}-2$ & 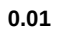 & & .03 \\
\hline 5 lin. & $1.1 \mathrm{E}+4$ & & $2.4 \mathrm{E}+3$ & $2.1 \mathrm{E}+1$ & $1.8 \mathrm{E}+1$ & $3.9 \mathrm{E}+1$ & 1 & & .02 \\
\hline ros. 10 lin. & 2.2 & & $1.8 \mathrm{E}+5$ & +2 & $8.4 \mathrm{E}+2$ & $2.5 \mathrm{E}+3$ & 2 & .02 & .04 \\
\hline ros. 20 lin. & 4.2 & & +7 & 5 & 6 & $E+6$ & & & .14 \\
\hline 50 lin. & $7.2 \mathrm{E}+9$ & 2.7E+8 & $5.2 E+8$ & $6.7 \mathrm{E}+9$ & $1.2 \mathrm{E}+8$ & $3.1 \mathrm{E}+8$ & 7 & 7 & 0.50 \\
\hline ros. 100 lin. & 4.6E+11 & $1.4 \mathrm{E}+10$ & $1.7 \mathrm{E}+10$ & 4.5E+11 & $1.1 \mathrm{E}+10$ & 1.3E+10 & 0.90 & 2 & 0.71 \\
\hline $2 \sin$. & $3.1 \mathrm{E}+6$ & $3.7 \mathrm{E}+4$ & $4.4 \mathrm{E}+1$ & $2.1 \mathrm{E}+6$ & $5 \mathrm{E}-1$ & $6.3 \mathrm{E}+0$ & 3 & 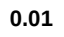 & .05 \\
\hline $5 \sin$. & $1.3 \mathrm{E}+9$ & $7.6 \mathrm{E}+3$ & $1.7 \mathrm{E}+4$ & $1.2 \mathrm{E}+9$ & $8.3 E+1$ & $4.1 \mathrm{E}+2$ & 5 &  & 0.04 \\
\hline ros. $10 \mathrm{sin}$. & $1.6 \mathrm{E}+6$ & $4.8 \mathrm{E}+5$ & $1.4 \mathrm{E}+6$ & $4.6 \mathrm{E}+3$ & $7.3 \mathrm{E}+3$ & $4.1 \mathrm{E}+4$ & 0.01 & 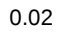 & 0.04 \\
\hline $20 \mathrm{sin}$. & $6.7 \mathrm{E}+6$ & $8.1 \mathrm{E}+6$ & $2.0 \mathrm{E}+7$ & $3.8 \mathrm{E}+5$ & $6.5 \mathrm{E}+5$ & $1.9 \mathrm{E}+6$ & 4 & 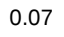 & 0.11 \\
\hline $50 \mathrm{sin}$. & $1.6 \mathrm{E}+9$ & $8.2 E+8$ & $2.0 \mathrm{E}+9$ & $1.4 \mathrm{E}+9$ & $3.2 \mathrm{E}+8$ & $9.1 \mathrm{E}+8$ & 5 & 7 & 0.37 \\
\hline ros. $100 \mathrm{sin}$. & $3.9 \mathrm{E}+11$ & $5.6 \mathrm{E}+9$ & $1.2 \mathrm{E}+10$ & $3.9 \mathrm{E}+11$ & $3.9 \mathrm{E}+9$ & $8.1 \mathrm{E}+9$ & .91 & 2 & 0.61 \\
\hline 2 lin. & $3.8 \mathrm{E}+0$ & $1.6 \mathrm{E}+0$ & $1.4 \mathrm{E}+0$ & $2 \mathrm{E}-2$ & $5.6 \mathrm{E}-3$ & $2.2 \mathrm{E}-2$ & 0.03 & 0.03 & 0.07 \\
\hline 5 lin. & $2.4 \mathrm{E}+5$ & 2.2E+1 & $2.7 \mathrm{E}+1$ & $2.4 \mathrm{E}+5$ & 7.4E+0 & $1.1 \mathrm{E}+1$ & .64 & .18 & 0.26 \\
\hline rast. 10 lin. & $5.9 \mathrm{E}+5$ & $9.9 \mathrm{E}+1$ & $1.3 \mathrm{E}+2$ & $5.9 \mathrm{E}+5$ & $5.6 \mathrm{E}+1$ & $6.8 \mathrm{E}+1$ & 0.90 & .33 & 0.36 \\
\hline rast. 20 lin. & $3.0 \mathrm{E}+5$ & $4.0 \mathrm{E}+2$ & $5.6 \mathrm{E}+2$ & $3.0 \mathrm{E}+5$ & $2.3 \mathrm{E}+2$ & $3.1 \mathrm{E}+2$ & 0.87 & .42 & 0.46 \\
\hline rast. 50 lin. & $2.0 \mathrm{E}+5$ & 4.1E+3 & $7.2 \mathrm{E}+3$ & $2.0 \mathrm{E}+5$ & $2.8 \mathrm{E}+3$ & $5.2 \mathrm{E}+3$ & 0.93 & 0.60 & 0.67 \\
\hline rast. 100 lin. & 3.2E+6 & $6.2 E+4$ & $6.2 E+4$ & $3.2 \mathrm{E}+6$ & $5.5 E+4$ & 5.3E+4 & 0.98 & 1 & 0.80 \\
\hline $2 \sin$. & $9.9 \mathrm{E}+1$ & $5.1 \mathrm{E}+0$ & $2.3 \mathrm{E}+0$ & $6.4 \mathrm{E}+1$ & -1 & E-1 & .18 & .04 & 0.09 \\
\hline $5 \sin$. & $4.7 \mathrm{E}+4$ & 2.6E+1 & $3.6 \mathrm{E}+1$ & $4.7 \mathrm{E}+4$ & $1.0 \mathrm{E}+1$ & $1.5 \mathrm{E}+1$ & 0.83 & .22 & 0.25 \\
\hline rast. $10 \mathrm{sin}$. & $1.3 \mathrm{E}+3$ & $1.8 \mathrm{E}+2$ & $2.6 \mathrm{E}+2$ & $1.1 \mathrm{E}+3$ & $7.5 \mathrm{E}+1$ & $1.0 \mathrm{E}+2$ & 0.41 & 0.17 & 0.19 \\
\hline rast. $20 \mathrm{sin}$. & $6.9 \mathrm{E}+4$ & $6.6 \mathrm{E}+2$ & $1.0 \mathrm{E}+3$ & $6.8 \mathrm{E}+4$ & $3.1 \mathrm{E}+2$ & $4.5 \mathrm{E}+2$ & 0.88 & 0.28 & 0.33 \\
\hline rast. $\quad 50 \mathrm{sin}$. & $1.8 \mathrm{E}+5$ & $1.0 \mathrm{E}+4$ & $1.5 \mathrm{E}+4$ & $1.7 \mathrm{E}+5$ & $6.2 \mathrm{E}+3$ & $9.6 \mathrm{E}+3$ & 0.89 & 0.49 & 0.56 \\
\hline rast. $100 \mathrm{sin}$. & $2.1 \mathrm{E}+5$ & 4.3E+4 & $5.1 \mathrm{E}+4$ & $2.1 \mathrm{E}+5$ & $3.5 \mathrm{E}+4$ & $4.2 \mathrm{E}+4$ & 0.93 & 0.71 & 0.73 \\
\hline Best frequen. & 3 & 25 & 8] & 8 & 26 & 2 & 11 & 21 & \\
\hline
\end{tabular}


Table 2: Results for pred2p on SRR benchmark

\begin{tabular}{|c|c|c|c|c|c|c|c|c|c|}
\hline \multirow{2}{*}{\begin{tabular}{|l|} 
Metric \\
Benchmark \\
\end{tabular}} & \multicolumn{3}{|c|}{ BOG } & \multicolumn{3}{|c|}{ BEBC } & \multicolumn{3}{|c|}{$\mathrm{RCS}$} \\
\hline & 5 & 49 & 0 & 5 & 9 & 0 & 5 & 9 & .00 \\
\hline sph. & -1 & $6.4 \mathrm{E}-2$ & $5.5 \mathrm{E}-2$ & 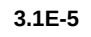 & -5 & $E-5$ & .01 & 1 & 0.03 \\
\hline 5 lin. & $8.0 \mathrm{E}+0$ & $3.0 \mathrm{E}+0$ & $4.2 \mathrm{E}+0$ & $1.9 \mathrm{E}-2$ & $2.8 \mathrm{E}-2$ & $7.1 \mathrm{E}-2$ & 0.02 & 0.02 & .047 \\
\hline ph. 10 lin. & & $2.2 \mathrm{E}+1$ & $9 \mathrm{E}+1$ & .1E-1 & $1.3 \mathrm{E}+0$ & $4.1 \mathrm{E}+0$ & .05 & .07 & 2 \\
\hline ph. 20 lin. & $E+2$ & $1.6 \mathrm{E}+2$ & $3.0 \mathrm{E}+2$ & $1.7 \mathrm{E}+1$ & $3.6 \mathrm{E}+1$ & $9.2 \mathrm{E}+1$ & .14 & .20 & .31 \\
\hline sph. 50 lin. & $2.7 E+3$ & $3.4 \mathrm{E}+3$ & $6.2 \mathrm{E}+3$ & $1.8 \mathrm{E}+3$ & $2.2 \mathrm{E}+3$ & $4.3 \mathrm{E}+3$ & 15 & 56 & 0.62 \\
\hline sph. 100 lin. & $6.4 \mathrm{E}+4$ & $5.5 E+4$ & $6.2 \mathrm{E}+4$ & $5.8 \mathrm{E}+4$ & 4.8E+4 & $5.3 E+4$ & 18 & 99 & .80 \\
\hline sph. & $7.3 \mathrm{E}+0$ & $5 \mathrm{E}-1$ & $4 \mathrm{E}-1$ & $5 E-5$ & $1.1 \mathrm{E}-4$ & $1.5 \mathrm{E}-4$ & .01 & 02 & .02 \\
\hline $5 \sin$. & $1.2 \mathrm{E}+1$ & $2.8 \mathrm{E}+0$ & $.0 \mathrm{E}+0$ & $7.1 \mathrm{E}-2$ & 3.8E-2 & $1.9 \mathrm{E}-1$ & .02 & 4 & .05 \\
\hline sph. $10 \mathrm{sin}$. & $1.5 \mathrm{E}+2$ & $9.3 \mathrm{E}+1$ & $1.7 \mathrm{E}+2$ & $4.0 \mathrm{E}+0$ & $5.5 \mathrm{E}+0$ & $1.8 \mathrm{E}+1$ & .05 & 6 & .10 \\
\hline sph. 20 sin. & $6.0 \mathrm{E}+2$ & $4.5 \mathrm{E}+2$ & $8.1 \mathrm{E}+2$ & $2.5 \mathrm{E}+2$ & $1.0 \mathrm{E}+2$ & $2.4 \mathrm{E}+2$ & .17 & 8 & .27 \\
\hline sph. $\quad 50$ sin. & $8.0 \mathrm{E}+3$ & $9.2 \mathrm{E}+3$ & $1.4 \mathrm{E}+4$ & $5.2 E+3$ & $5.5 E+3$ & $9.2 \mathrm{E}+3$ & .20 & 7 & .55 \\
\hline sph. $100 \mathrm{sin}$. & $3.8 \mathrm{E}+4$ & $4.1 \mathrm{E}+4$ & $5.1 \mathrm{E}+4$ & $3.2 \mathrm{E}+4$ & $3.3 E+4$ & $4.2 \mathrm{E}+4$ & 25 & & 0.73 \\
\hline 2 lin. & $3.8 \mathrm{E}+2$ & $E+0$ & $1.6 \mathrm{E}+0$ & $=-3$ & $4.2 \mathrm{E}-3$ & $1.1 \mathrm{E}-2$ & 1 & & .03 \\
\hline 5 lin. & $1.1 \mathrm{E}+4$ & & $2.4 \mathrm{E}+3$ & $2.0 \mathrm{E}+1$ & $2.0 \mathrm{E}+1$ & $3.7 \mathrm{E}+1$ & 1 & & .02 \\
\hline ros. 10 lin. & 2.2 & & $3.4 \mathrm{E}+5$ & +2 & $9.5 \mathrm{E}+2$ & $8.7 \mathrm{E}+4$ & 02 &  & .06 \\
\hline ros. 20 lin. & 2. & & +7 & -5 & 3 & $E+6$ & 5 & & .17 \\
\hline 50 lin. & $1.3 E+8$ & $2.6 \mathrm{E}+8$ & $5.5 \mathrm{E}+8$ & $4.8 \mathrm{E}+7$ & $1.1 \mathrm{E}+8$ & $3.2 \mathrm{E}+8$ & 20 & 6 & 0.52 \\
\hline ros. 100 lin. & $1.1 \mathrm{E}+10$ & $9.1 \mathrm{E}+9$ & $1.8 \mathrm{E}+10$ & $9.5 \mathrm{E}+9$ & $7.1 \mathrm{E}+9$ & $1.4 \mathrm{E}+10$ & 16 & 5 & 0.74 \\
\hline $2 \sin$. & $3.1 \mathrm{E}+5$ & $3.0 \mathrm{E}+4$ & $2.9 \mathrm{E}+1$ & $3.8 \mathrm{E}+3$ & $1.5 \mathrm{E}+0$ & $3.4 \mathrm{E}+0$ & 05 &  & 0.04 \\
\hline $5 \sin$. & $4.1 \mathrm{E}+5$ & $5.1 \mathrm{E}+3$ & $1.6 \mathrm{E}+4$ & $3.7 \mathrm{E}+1$ & $7.8 \mathrm{E}+1$ & $6.7 \mathrm{E}+2$ & 0.00 & - & 0.04 \\
\hline ros. $10 \mathrm{sin}$. & $1.6 \mathrm{E}+6$ & $5.6 \mathrm{E}+5$ & $1.4 \mathrm{E}+6$ & $5.2 \mathrm{E}+3$ & $1.2 \mathrm{E}+4$ & $3.6 \mathrm{E}+4$ & 0.01 & 2 & 0.04 \\
\hline $20 \mathrm{sin}$. & $5.3 E+6$ & $7.8 \mathrm{E}+6$ & $2.3 \mathrm{E}+7$ & $1.1 \mathrm{E}+5$ & $6.2 \mathrm{E}+5$ & $2.3 \mathrm{E}+6$ & 0.03 & 7 & 0.12 \\
\hline $50 \mathrm{sin}$. & $3.2 \mathrm{E}+8$ & $7.8 \mathrm{E}+8$ & $2.1 \mathrm{E}+9$ & $1.2 \mathrm{E}+8$ & $3.0 \mathrm{E}+8$ & $9.4 \mathrm{E}+8$ & 0.14 & 6 & 0.38 \\
\hline ros. $100 \mathrm{sin}$. & $3.5 \mathrm{E}+9$ & $5.5 \mathrm{E}+9$ & $1.1 \mathrm{E}+10$ & $2.5 \mathrm{E}+9$ & $3.7 \mathrm{E}+9$ & $8.0 \mathrm{E}+9$ & 0.16 & 0 & 0.60 \\
\hline 2 lin. & $3.8 \mathrm{E}+0$ & $1.7 \mathrm{E}+0$ & $1.4 \mathrm{E}+0$ & $1 \mathrm{E}-2$ & $6.6 \mathrm{E}-3$ & $2.6 \mathrm{E}-2$ & 0.03 & 3 & 0.07 \\
\hline 5 lin. & $4.9 \mathrm{E}+1$ & 2.2E+1 & $2.7 \mathrm{E}+1$ & $2.0 \mathrm{E}+1$ & $7.5 \mathrm{E}+0$ & $1.1 \mathrm{E}+1$ & 0.08 & .18 & 0.26 \\
\hline rast. 10 lin. & $2.2 \mathrm{E}+2$ & $1.0 \mathrm{E}+2$ & $1.3 \mathrm{E}+2$ & $1.3 \mathrm{E}+2$ & $5.6 \mathrm{E}+1$ & $6.8 \mathrm{E}+1$ & 0.08 & .33 & 0.36 \\
\hline rast. 20 lin. & $6.2 \mathrm{E}+2$ & $4.0 \mathrm{E}+2$ & $5.6 \mathrm{E}+2$ & $3.9 \mathrm{E}+2$ & $2.3 \mathrm{E}+2$ & $3.1 \mathrm{E}+2$ & 0.12 & .42 & 0.46 \\
\hline rast. 50 lin. & $6.7 \mathrm{E}+3$ & $4.0 \mathrm{E}+3$ & $7.0 \mathrm{E}+3$ & $5.4 \mathrm{E}+3$ & $2.8 \mathrm{E}+3$ & $5.0 \mathrm{E}+3$ & .17 & .60 & 0.65 \\
\hline rast. 100 lin. & $9.3 E+4$ & 5.3E+4 & $6.3 E+4$ & $8.6 E+4$ & 4.6E+4 & $5.4 \mathrm{E}+4$ & 1 & 71 & 0.81 \\
\hline $2 \sin$. & $3.2 \mathrm{E}+1$ & $5.2 \mathrm{E}+0$ & $2.3 \mathrm{E}+0$ & $1.1 \mathrm{E}+1$ & -1 & $\mathrm{E}-1$ & 0.06 & .04 & 0.09 \\
\hline $5 \sin$. & $1.0 \mathrm{E}+2$ & 2.6E+1 & $3.5 \mathrm{E}+1$ & $6.2 \mathrm{E}+1$ & $1.0 \mathrm{E}+1$ & $1.5 \mathrm{E}+1$ & 0.07 & .22 & 0.25 \\
\hline rast. $10 \mathrm{sin}$. & $4.1 \mathrm{E}+2$ & $1.8 \mathrm{E}+2$ & $2.6 \mathrm{E}+2$ & $2.2 \mathrm{E}+2$ & $7.5 \mathrm{E}+1$ & $9.9 \mathrm{E}+1$ & 0.16 & 0.17 & 0.19 \\
\hline rast. $20 \mathrm{sin}$. & $1.1 \mathrm{E}+3$ & $6.7 \mathrm{E}+2$ & $1.0 \mathrm{E}+3$ & $7.0 \mathrm{E}+2$ & $3.1 \mathrm{E}+2$ & $4.5 \mathrm{E}+2$ & 0.12 & 0.28 & 0.33 \\
\hline rast. $\quad 50 \mathrm{sin}$. & $9.8 \mathrm{E}+3$ & $1.0 \mathrm{E}+4$ & $1.5 \mathrm{E}+4$ & $7.0 \mathrm{E}+3$ & $6.1 \mathrm{E}+3$ & $9.6 \mathrm{E}+3$ & 0.18 & 0.49 & 0.56 \\
\hline rast. $100 \mathrm{sin}$. & $4.0 \mathrm{E}+4$ & 4.3E+4 & $5.2 \mathrm{E}+4$ & $3.4 \mathrm{E}+4$ & $3.5 \mathrm{E}+4$ & $4.2 \mathrm{E}+4$ & 0.29 & 0.71 & 0.73 \\
\hline Best frequen. & 11 & 18 & 7 & 18 & 18 & 0 & 33 & 3 & \\
\hline
\end{tabular}


Table 3: Results for pred3 on SRR benchmark

\begin{tabular}{|c|c|c|c|c|c|c|c|c|c|c|c|c|c|c|c|}
\hline \multirow{2}{*}{\begin{tabular}{|l|} 
Metric \\
Benchmark \\
\end{tabular}} & \multicolumn{5}{|c|}{ BOG } & \multicolumn{5}{|c|}{ BEBC } & \multicolumn{5}{|c|}{$\mathrm{RCS}$} \\
\hline & 1.05 & 1.49 & 2.00 & additive & adaptive & 1.05 & 1.49 & 2.00 & additive & adaptive & 1.05 & 1.49 & 2.00 & additive & aptive \\
\hline 2 lin. & $5.0 \mathrm{E}-1$ & $4.8 \mathrm{E}-2$ & $5.7 \mathrm{E}-2$ & 4.1E-2 & $4.8 \mathrm{E}-2$ & $2.8 \mathrm{E}-5$ & $5.2 \mathrm{E}-5$ & $6.3 E-5$ & $5.4 \mathrm{E}-5$ & $5.1 \mathrm{E}-5$ & 0.00 & 0.01 & 0.03 & 0.04 & 0.02 \\
\hline 5 lin. & $3.1 \mathrm{E}+0$ & $2.1 \mathrm{E}+0$ & $3.4 \mathrm{E}+0$ & $2.4 \mathrm{E}+0$ & $2.1 \mathrm{E}+0$ & $1.8 \mathrm{E}-2$ & $2.6 \mathrm{E}-2$ & $6.3 \mathrm{E}-2$ & $3.2 \mathrm{E}-2$ & 2.7E-2 & 0.01 & 0.02 & 0.04 & 0.06 & 0.04 \\
\hline sph. $\quad 10$ lin. & $1.5 \mathrm{E}+1$ & 1.7E+1 & $3.1 \mathrm{E}+1$ & $2.1 \mathrm{E}+1$ & $1.7 \mathrm{E}+1$ & 3.2E-1 & 1.3E+0 & $3.3 \mathrm{E}+0$ & $1.8 \mathrm{E}+0$ & $1.3 \mathrm{E}+0$ & 0.02 & 0.06 & 0.09 & .11 & .10 \\
\hline sph. 20 lin. & 7.7E+1 & $1.4 \mathrm{E}+2$ & $2.4 \mathrm{E}+2$ & $1.4 \mathrm{E}+2$ & $1.4 \mathrm{E}+2$ & $9.6 \mathrm{E}+0$ & $3.5 \mathrm{E}+1$ & $6.9 \mathrm{E}+1$ & $3.1 \mathrm{E}+1$ & $3.5 E+1$ & 0.07 & 0.18 & .24 & .22 & .26 \\
\hline sph. $\quad 50$ lin. & $1.7 \mathrm{E}+3$ & $2.2 \mathrm{E}+3$ & $5.7 \mathrm{E}+3$ & $2.5 \mathrm{E}+3$ & $2.2 \mathrm{E}+3$ & $1.1 \mathrm{E}+3$ & $1.4 \mathrm{E}+3$ & $3.9 \mathrm{E}+3$ & $1.5 \mathrm{E}+3$ & $1.4 \mathrm{E}+3$ & 0.13 & 0.34 & 0.57 & 0.49 & 0.51 \\
\hline sph. $100 \mathrm{lin}$. & $4.0 \mathrm{E}+4$ & $2.8 \mathrm{E}+4$ & $5.1 \mathrm{E}+4$ & $2.8 \mathrm{E}+4$ & $2.7 E+4$ & $3.6 \mathrm{E}+4$ & 2.3E+4 & 4. $3 E+4$ & $2.3 \mathrm{E}+4$ & $2.2 \mathrm{E}+4$ & 0.16 & 0.37 & 0.65 & 0.72 & 0.70 \\
\hline $2 \sin$. & $2.1 \mathrm{E}+0$ & $1.1 \mathrm{E}-1$ & $0 \mathrm{E}-1$ & 7E-1 & $1 \mathrm{E}-1$ & $7.9 \mathrm{E}-5$ & $1.6 \mathrm{E}-4$ & $2 \mathrm{E}-4$ & $4 \mathrm{E}-4$ & $6 \mathrm{E}-4$ & 0.00 & 0.02 & .03 & .03 & 0.03 \\
\hline $5 \sin$. & $5.0 \mathrm{E}+0$ & $3.8 \mathrm{E}+0$ & $9.6 \mathrm{E}+0$ & $6.2 \mathrm{E}+0$ & $3.8 \mathrm{E}+0$ & 3.4E-2 & $5.9 \mathrm{E}-2$ & $2.4 \mathrm{E}-1$ & $9.7 \mathrm{E}-2$ & $5.9 \mathrm{E}-2$ & 0.02 & 0.05 & .07 & .06 & .06 \\
\hline sph. $10 \mathrm{sin}$. & $1.1 \mathrm{E}+2$ & 1.1E+2 & $1.9 \mathrm{E}+2$ & 1.3E+2 & $1.1 \mathrm{E}+2$ & 2.4E+0 & $6.6 \mathrm{E}+0$ & $1.8 \mathrm{E}+1$ & $7.5 \mathrm{E}+0$ & $E+0$ & 0.03 & 0.07 & .11 & .08 & .09 \\
\hline sph. 20 sin. & 4.4E+2 & $4.6 \mathrm{E}+2$ & $8.1 \mathrm{E}+2$ & $4.9 E+2$ & $4.6 \mathrm{E}+2$ & $1.6 \mathrm{E}+2$ & $1.1 \mathrm{E}+2$ & $2.3 E+2$ & $1.1 \mathrm{E}+2$ & $1.1 \mathrm{E}+2$ & 0.12 & 0.19 & .26 & 20 & .20 \\
\hline sph. $\quad 50 \sin$. & $5.6 \mathrm{E}+3$ & $8.2 E+3$ & $1.5 \mathrm{E}+4$ & $9.1 E+3$ & $8.2 \mathrm{E}+3$ & $2.9 E+3$ & $4.5 E+3$ & $.2 E+3$ & $.2 E+3$ & $4.5 E+3$ & 0.15 & 42 & .56 & 48 & 40 \\
\hline sph. $100 \mathrm{sin}$. & $2.8 \mathrm{E}+4$ & $3.7 \mathrm{E}+4$ & $5.2 \mathrm{E}+4$ & $3.7 E+4$ & $3.7 \mathrm{E}+4$ & $2.1 \mathrm{E}+4$ & $2.8 \mathrm{E}+4$ & $4.2 \mathrm{E}+4$ & $2.8 \mathrm{E}+4$ & $2.8 \mathrm{E}+4$ & 0.20 & 62 & .73 & 0 & 0.68 \\
\hline 2 lin. & $1.2 \mathrm{E}+2$ & $7.8 \mathrm{E}-1$ & $1.6 \mathrm{E}+0$ & $E-1$ & $9 \mathrm{E}-1$ & $5.5 \mathrm{E}-3$ & OE-3 & $2 \mathrm{E}-2$ & $2 \mathrm{E}-3$ & 2E-3 & .00 & & 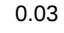 & & .02 \\
\hline 5 lin. & $5.4 \mathrm{E}+3$ & $2.1 \mathrm{E}+3$ & $2.4 \mathrm{E}+3$ & $2.2 \mathrm{E}+3$ & $2.1 \mathrm{E}+3$ & 1.9E+1 & $1.9 \mathrm{E}+1$ & $3.5 \mathrm{E}+1$ & $2.1 \mathrm{E}+1$ & $2.0 \mathrm{E}+1$ & .00 & 01 & 02 & 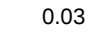 & .03 \\
\hline ros. $10 \mathrm{lin}$. & $1.9 \mathrm{E}+5$ & $8.4 \mathrm{E}+4$ & $3.8 \mathrm{E}+5$ & $9.0 \mathrm{E}+4$ & $8.3 E+4$ & 1.2E+2 & $6.3 \mathrm{E}+2$ & $8.1 \mathrm{E}+4$ & $6.7 \mathrm{E}+2$ & $5.8 \mathrm{E}+2$ & 01 & 2 & 05 & 4 & .04 \\
\hline ros. 20 lin. & $2.2 \mathrm{E}+6$ & $1.1 \mathrm{E}+7$ & +7 & $9.5 \mathrm{E}+6$ & $1.1 \mathrm{E}+7$ & $4.9 E+3$ & $E+6$ & +6 & $2.5 \mathrm{E}+6$ & $3.0 \mathrm{E}+6$ & 1 & 0.17 & .14 & (1. & 0.19 \\
\hline ros. 50 lin. & & $1.4 \mathrm{E}+8$ & $E+8$ & 1.7 & $1.4 \mathrm{E}+8$ & 1. & +7 & & $E+7$ & $5.1 \mathrm{E}+7$ & 09 & 9 & 39 & .33 & .32 \\
\hline ros. 100 lin. & 7.7E+9 & 3.8E+9 & $1.1 \mathrm{E}+10$ & $3.5 \mathrm{E}+9$ & $3.6 E+9$ & $6.8 \mathrm{E}+9$ & 2.7E+9 & $8.5 E+9$ & $2.5 \mathrm{E}+9$ & $2.5 E+9$ & 14 & 0.26 & .50 & $=-$ & 0.59 \\
\hline $2 \sin$. & $5.9 \mathrm{E}+4$ & $6.2 \mathrm{E}+3$ & $2.9 \mathrm{E}+1$ & $6.2 \mathrm{E}+3$ & $6.3 \mathrm{E}+3$ & $3.7 \mathrm{E}+3$ & 1.3E-1 & $1.5 \mathrm{E}+0$ & E-1 & $E-1$ & - & &  & & . \\
\hline $5 \sin$. & $1.2 \mathrm{E}+5$ & $5.4 \mathrm{E}+3$ & $2.8 \mathrm{E}+4$ & $1.3 \mathrm{E}+4$ & $5.4 \mathrm{E}+3$ & $1.1 \mathrm{E}+1$ & $9.8 \mathrm{E}+1$ & 7.7E+2 & $1.9 \mathrm{E}+2$ & $9.7 \mathrm{E}+1$ & 0.00 & &  & & .04 \\
\hline $10 \mathrm{sin}$. & $9.1 \mathrm{E}+5$ & $6.4 \mathrm{E}+5$ & $1.9 \mathrm{E}+6$ & $8.9 E+5$ & $6.3 E+5$ & $2.9 E+3$ & 1.3E+4 & $4.2 \mathrm{E}+4$ & $1.6 \mathrm{E}+4$ & $1.3 E+4$ & 1 & 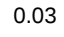 & 0.04 & & .03 \\
\hline $20 \sin$. & $3.6 \mathrm{E}+6$ & $8.4 \mathrm{E}+6$ & $2.4 \mathrm{E}+7$ & $1.0 \mathrm{E}+7$ & $8.4 \mathrm{E}+6$ & $5.7 \mathrm{E}+4$ & $6.7 \mathrm{E}+5$ & $2.2 \mathrm{E}+6$ & $6.7 \mathrm{E}+5$ & $6.5 \mathrm{E}+5$ & 0.02 & 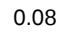 & - & & 0.09 \\
\hline $50 \sin$. & $2.4 \mathrm{E}+8$ & $6.4 \mathrm{E}+8$ & $2.0 \mathrm{E}+9$ & $7.9 \mathrm{E}+8$ & $6.5 \mathrm{E}+8$ & $6.7 \mathrm{E}+7$ & $2.2 \mathrm{E}+8$ & $8.6 \mathrm{E}+8$ & $2.8 \mathrm{E}+8$ & $2.2 \mathrm{E}+8$ & 0.10 & 0. & 36 & 0.27 & .26 \\
\hline ros. $100 \mathrm{sin}$. & $2.6 \mathrm{E}+9$ & $5.1 \mathrm{E}+9$ & $1.1 \mathrm{E}+10$ & $5.2 \mathrm{E}+9$ & $5.1 \mathrm{E}+9$ & $1.5 \mathrm{E}+9$ & $3.2 \mathrm{E}+9$ & 7.3E+9 & 3. $3 \mathrm{E}+9$ & $3.2 \mathrm{E}+9$ & 0.14 & 0.46 & .56 & 0.51 & 0.52 \\
\hline 2 lin. & $2.1 \mathrm{E}+0$ & $1.2 \mathrm{E}+0$ & $E+0$ & 1.1E+0 & $1.2 \mathrm{E}+0$ & 4.7E-3 & $6.1 \mathrm{E}-3$ & $2.9 \mathrm{E}-2$ & $9 \mathrm{E}-3$ & $5.7 \mathrm{E}-3$ & 1 & 0.02 & 7 & 7 & 0.04 \\
\hline $5 \mathrm{lin}$. & $2.2 \mathrm{E}+1$ & $2.0 \mathrm{E}+1$ & $2.6 \mathrm{E}+1$ & $2.2 \mathrm{E}+1$ & $2.0 \mathrm{E}+1$ & $6.9 E+0$ & $7.2 \mathrm{E}+0$ & $1.1 \mathrm{E}+1$ & $8.8 \mathrm{E}+0$ & $7.3 E+0$ & 0.05 & 0.17 & .25 & 9 & 0.26 \\
\hline rast. $10 \mathrm{lin}$. & $1.3 \mathrm{E}+2$ & $9.5 E+1$ & $1.2 \mathrm{E}+2$ & $9.9 \mathrm{E}+1$ & $9.5 E+1$ & $8.1 E+1$ & $5.6 \mathrm{E}+1$ & $6.4 \mathrm{E}+1$ & $5.7 \mathrm{E}+1$ & $5.6 \mathrm{E}+1$ & 0.08 & 0.33 & 0.33 & 0.39 & 0.41 \\
\hline rast. 20 lin. & $3.9 E+2$ & $3.6 \mathrm{E}+2$ & $5.0 \mathrm{E}+2$ & $3.7 \mathrm{E}+2$ & $3.6 \mathrm{E}+2$ & $2.5 E+2$ & $2.2 \mathrm{E}+2$ & $2.9 \mathrm{E}+2$ & $2.2 \mathrm{E}+2$ & $2.2 \mathrm{E}+2$ & 0.10 & 0.39 & 0.41 & .44 & 0.48 \\
\hline rast. 50 lin. & $4.9 \mathrm{E}+3$ & $2.6 \mathrm{E}+3$ & $6.4 \mathrm{E}+3$ & $3.0 \mathrm{E}+3$ & $2.6 \mathrm{E}+3$ & $4.1 \mathrm{E}+3$ & $1.8 \mathrm{E}+3$ & $4.6 \mathrm{E}+3$ & $2.0 \mathrm{E}+3$ & $1.8 \mathrm{E}+3$ & 0.15 & 0.39 & 0.59 & 0.55 & 0.58 \\
\hline rast. 100 lin. & $5.3 E+4$ & $2.9 E+4$ & $5.1 \mathrm{E}+4$ & $3.0 E+4$ & $2.9 E+4$ & $4.8 E+4$ & $2.4 \mathrm{E}+4$ & 4.3E+4 & $2.4 \mathrm{E}+4$ & $2.4 E+4$ & 0.17 & 0.39 & 0.66 & 0.72 & 0.71 \\
\hline $2 \sin$. & $1.7 \mathrm{E}+1$ & $2.4 \mathrm{E}+0$ & $2.7 \mathrm{E}+0$ & $2.7 \mathrm{E}+0$ & $2.4 \mathrm{E}+0$ & $9.4 \mathrm{E}+0$ & 8.5E-2 & $2.0 \mathrm{E}-1$ & 1.3E-1 & 8.5E-2 & 0.04 & 0.04 & .10 & .09 & .09 \\
\hline $5 \sin$. & $4.6 \mathrm{E}+1$ & $2.8 \mathrm{E}+1$ & $4.0 \mathrm{E}+1$ & $3.3 E+1$ & $2.8 \mathrm{E}+1$ & $2.5 E+1$ & 1.1E+1 & $1.6 \mathrm{E}+1$ & 1.3E+1 & $1.1 \mathrm{E}+1$ & 0.06 & 0.25 & .27 & .27 & .29 \\
\hline rast. $10 \mathrm{sin}$. & $3.1 \mathrm{E}+2$ & $2.0 \mathrm{E}+2$ & $2.8 \mathrm{E}+2$ & $2.2 \mathrm{E}+2$ & $2.0 \mathrm{E}+2$ & $1.6 \mathrm{E}+2$ & 7.9E+1 & $1.0 \mathrm{E}+2$ & $8.0 E+1$ & $7.9 \mathrm{E}+1$ & 0.12 & 0.18 & .20 & .18 & 0.20 \\
\hline rast. 20 sin. & $8.0 \mathrm{E}+2$ & $6.8 \mathrm{E}+2$ & $1.0 \mathrm{E}+3$ & $7.2 \mathrm{E}+2$ & $6.8 \mathrm{E}+2$ & $4.8 \mathrm{E}+2$ & $3.1 \mathrm{E}+2$ & 4.4E+2 & $3.1 \mathrm{E}+2$ & $3.1 \mathrm{E}+2$ & 0.10 & 0.28 & 0.33 & 0.28 & 0.30 \\
\hline rast. $50 \mathrm{sin}$. & $6.7 \mathrm{E}+3$ & $8.8 E+3$ & $1.5 \mathrm{E}+4$ & $9.8 \mathrm{E}+3$ & $8.9 E+3$ & $4.0 \mathrm{E}+3$ & $5.1 \mathrm{E}+3$ & $9.4 \mathrm{E}+3$ & $5.8 \mathrm{E}+3$ & $5.1 \mathrm{E}+3$ & 0.14 & 0.43 & 0.56 & 0.49 & 0.48 \\
\hline rast. $100 \mathrm{sin}$. & $2.9 \mathrm{E}+4$ & $3.9 \mathrm{E}+4$ & $5.2 \mathrm{E}+4$ & $3.9 \mathrm{E}+4$ & $3.9 \mathrm{E}+4$ & $2.2 \mathrm{E}+4$ & $3.0 \mathrm{E}+4$ & 4.2E+4 & $3.0 \mathrm{E}+4$ & $3.0 \mathrm{E}+4$ & 0.23 & 0.63 & 0.74 & 0.69 & 0.69 \\
\hline Best frequen. & 13 & 12 & 1 & 4 & 6 & 23 & 7 & 0 & 3 & - & 35 & 1 & 0 & 0 & \\
\hline
\end{tabular}


Table 4: Results for pred3p on SRR benchmark

\begin{tabular}{|c|c|c|c|c|c|c|c|c|c|c|c|c|c|c|c|}
\hline \multirow{2}{*}{\begin{tabular}{|l|} 
Metric \\
Benchmark \\
\end{tabular}} & \multicolumn{5}{|c|}{ BOG } & \multicolumn{5}{|c|}{ BEBC } & \multicolumn{5}{|c|}{ RCS } \\
\hline & 1.05 & 1.49 & 2.00 & idditive & adaptive & 1.05 & 1.49 & 2.00 & dditive & adaptive & 1.05 & 1.49 & 2.00 & additive & apive \\
\hline 2 lin. & 5.0E-1 & $4.8 \mathrm{E}-2$ & 5.7E-2 & 4.2E-2 & $4.9 \mathrm{E}-2$ & 2.9E-5 & 5.1E-5 & $6.7 \mathrm{E}-5$ & $5.3 E-5$ & $5.3 \mathrm{E}-5$ & 0.00 & 0.01 & 0.03 & 0.04 & . \\
\hline $5 \mathrm{lin}$. & $3.1 \mathrm{E}+0$ & $2.1 \mathrm{E}+0$ & $3.4 \mathrm{E}+0$ & $2.4 \mathrm{E}+0$ & $2.1 \mathrm{E}+0$ & $1.8 \mathrm{E}-2$ & 2.7E-2 & $6.5 \mathrm{E}-2$ & $3.2 \mathrm{E}-2$ & $2.7 \mathrm{E}-2$ & 0.01 & 0.02 & 0.04 & .06 & S \\
\hline sph. 10 lin. & $1.5 \mathrm{E}+1$ & $1.7 \mathrm{E}+1$ & $3.1 \mathrm{E}+1$ & $2.1 \mathrm{E}+1$ & $1.7 \mathrm{E}+1$ & 3.2E-1 & $1.3 \mathrm{E}+0$ & $3.3 \mathrm{E}+0$ & 1.7E+0 & $1.3 \mathrm{E}+0$ & 0.02 & 0.06 & 0.09 & .11 & .10 \\
\hline sph. 20 lin. & 7.7E+1 & $1.4 \mathrm{E}+2$ & $2.4 \mathrm{E}+2$ & $1.4 \mathrm{E}+2$ & $1.4 \mathrm{E}+2$ & $9.6 \mathrm{E}+0$ & $3.5 \mathrm{E}+1$ & $6.9 \mathrm{E}+1$ & $3.0 E+1$ & $3.4 \mathrm{E}+1$ & .07 & 0.18 & .24 & .21 & .26 \\
\hline sph. 50 lin. & $1.7 \mathrm{E}+3$ & $2.2 \mathrm{E}+3$ & $5.7 \mathrm{E}+3$ & $2.5 E+3$ & $2.2 \mathrm{E}+3$ & $1.2 \mathrm{E}+3$ & $1.4 \mathrm{E}+3$ & $3.9 \mathrm{E}+3$ & $1.5 \mathrm{E}+3$ & $1.3 \mathrm{E}+3$ & .13 & 0.34 & .57 & .50 & .50 \\
\hline sph. 100 lin. & $4.2 E+4$ & $2.9 \mathrm{E}+4$ & $5.2 E+4$ & $2.8 E+4$ & $2.8 E+4$ & $3.7 E+4$ & $2.4 \mathrm{E}+4$ & 4.4E+4 & 2.3E+4 & $2.3 E+4$ & 0.17 & 0.38 & 0.67 & .71 & 0.73 \\
\hline $2 \sin$. & $2.1 \mathrm{E}+0$ & $1.1 \mathrm{E}-1$ & 2.0E-1 & 1.7E-1 & $1.2 \mathrm{E}-1$ & 8.0E-5 & 1.6E-4 & 2.3E-4 & $2.5 \mathrm{E}-4$ & $1.6 \mathrm{E}-4$ & 0.00 & 0.02 & 0.03 & 0.03 & 0.03 \\
\hline $5 \sin$. & $5.0 \mathrm{E}+0$ & $3.8 \mathrm{E}+0$ & $9.5 \mathrm{E}+0$ & $6.2 \mathrm{E}+0$ & $3.8 \mathrm{E}+0$ & $3.4 \mathrm{E}-2$ & $5.9 \mathrm{E}-2$ & $2.4 \mathrm{E}-1$ & $9.8 \mathrm{E}-2$ & $5.9 \mathrm{E}-2$ & 0.02 & 0.05 & 0.07 & 0.06 & 0.06 \\
\hline sph. 10 sin. & $1.1 \mathrm{E}+2$ & $1.1 \mathrm{E}+2$ & $1.9 \mathrm{E}+2$ & $1.3 \mathrm{E}+2$ & $1.1 \mathrm{E}+2$ & $2.4 \mathrm{E}+0$ & $6.7 \mathrm{E}+0$ & $1.8 \mathrm{E}+1$ & $7.3 \mathrm{E}+0$ & $6.7 \mathrm{E}+0$ & 0.03 & 0.07 & 0.11 & 0.08 & 0.08 \\
\hline sph. $20 \mathrm{sin}$. & $4.4 \mathrm{E}+2$ & $4.6 \mathrm{E}+2$ & $8.2 \mathrm{E}+2$ & $5.0 \mathrm{E}+2$ & $4.6 \mathrm{E}+2$ & $1.6 \mathrm{E}+2$ & $1.1 \mathrm{E}+2$ & $2.3 E+2$ & $1.1 \mathrm{E}+2$ & $1.1 \mathrm{E}+2$ & 0.12 & 0.19 & 0.26 & 0.19 & 0.20 \\
\hline sph. $\quad 50$ sin. & $5.6 \mathrm{E}+3$ & $8.2 \mathrm{E}+3$ & $1.5 \mathrm{E}+4$ & $9.1 \mathrm{E}+3$ & $8.2 \mathrm{E}+3$ & $2.9 \mathrm{E}+3$ & $4.5 \mathrm{E}+3$ & $9.0 \mathrm{E}+3$ & $5.2 \mathrm{E}+3$ & $4.5 \mathrm{E}+3$ & .15 & .41 & .55 & 48 & 0.46 \\
\hline sph. $100 \mathrm{sin}$. & $2.7 \mathrm{E}+4$ & $3.6 \mathrm{E}+4$ & $5.2 \mathrm{E}+4$ & $3.6 \mathrm{E}+4$ & $3.6 \mathrm{E}+4$ & $2.0 \mathrm{E}+4$ & $2.8 \mathrm{E}+4$ & $4.2 \mathrm{E}+4$ & $2.8 \mathrm{E}+4$ & $2.8 \mathrm{E}+4$ & 0.20 & 0.62 & 0.73 & 0.67 & 0.68 \\
\hline 2 lin. & $1.2 \mathrm{E}+2$ & 7.7E-1 & $1.6 \mathrm{E}+0$ & 6.1E-1 & $7.8 \mathrm{E}-1$ & $5.5 \mathrm{E}-3$ & $9.9 \mathrm{E}-4$ & 1.7E-2 & $1.2 \mathrm{E}-3$ & $9.6 \mathrm{E}-4$ & 0.00 & 0.01 & 0.03 & 0.03 & 0.02 \\
\hline $5 \mathrm{lin}$. & $5.4 \mathrm{E}+3$ & $2.1 \mathrm{E}+3$ & $2.3 \mathrm{E}+3$ & $2.2 \mathrm{E}+3$ & $2.1 \mathrm{E}+3$ & $1.9 \mathrm{E}+1$ & $1.9 \mathrm{E}+1$ & $3.6 \mathrm{E}+1$ & $2.3 E+1$ & $1.9 \mathrm{E}+1$ & 0.00 & 0.01 & 0.02 & 0.03 & 0.02 \\
\hline ros. $10 \mathrm{lin}$. & $1.9 \mathrm{E}+5$ & $8.4 \mathrm{E}+4$ & $3.0 \mathrm{E}+5$ & $8.9 \mathrm{E}+4$ & $8.3 E+4$ & $1.2 \mathrm{E}+2$ & $7.3 \mathrm{E}+2$ & $4.5 \mathrm{E}+4$ & $6.1 \mathrm{E}+2$ & $5.6 \mathrm{E}+2$ & 0.01 & 0.02 & 0.05 & 0.04 & 0.04 \\
\hline ros. 20 lin. & $2.2 \mathrm{E}+6$ & $1.0 \mathrm{E}+7$ & $1.5 \mathrm{E}+7$ & $9.2 \mathrm{E}+6$ & $1.1 \mathrm{E}+7$ & $4.9 \mathrm{E}+3$ & $2.8 \mathrm{E}+6$ & $2.5 \mathrm{E}+6$ & $2.4 \mathrm{E}+6$ & $3.1 \mathrm{E}+6$ & 0.01 & 0.15 & 0.13 & 0.17 & 0.20 \\
\hline ros. 50 lin. & 7.1E+7 & $1.4 \mathrm{E}+8$ & $4.6 \mathrm{E}+8$ & $1.7 \mathrm{E}+8$ & $1.5 \mathrm{E}+8$ & $2.0 \mathrm{E}+7$ & $5.2 \mathrm{E}+7$ & $2.7 \mathrm{E}+8$ & $6.3 \mathrm{E}+7$ & $5.3 \mathrm{E}+7$ & 0.10 & 0.19 & 0.40 & 0.33 & 0.33 \\
\hline ros. 100 lin. & 7.7E+9 & 3.7E+9 & 1.1E+10 & 3.4E+9 & 3.7E+9 & $6.8 E+9$ & $2.7 \mathrm{E}+9$ & $8.5 E+9$ & 2.4E+9 & $2.6 E+9$ & - & 0.26 & 0.50 & 0.57 & 0.60 \\
\hline $2 \sin$. & $5.9 \mathrm{E}+4$ & $6.2 \mathrm{E}+3$ & 3.6E+1 & $6.2 \mathrm{E}+3$ & $6.2 \mathrm{E}+3$ & $3.7 \mathrm{E}+3$ & 1.3E-1 & $2.6 \mathrm{E}+0$ & $1.6 \mathrm{E}-1$ & $1.4 \mathrm{E}-1$ & 0.04 & 0.01 & 0.05 & 0.04 & 0.04 \\
\hline $5 \sin$. & $1.1 \mathrm{E}+5$ & $5.5 \mathrm{E}+3$ & $2.9 \mathrm{E}+4$ & $1.4 \mathrm{E}+4$ & $5.3 \mathrm{E}+3$ & $9.2 \mathrm{E}+0$ & $1.0 \mathrm{E}+2$ & $7.3 \mathrm{E}+2$ & $2.1 \mathrm{E}+2$ & $9.9 \mathrm{E}+1$ & 0.00 & 0.03 & 0.05 & 0.04 & 0.04 \\
\hline ros. $10 \mathrm{sin}$. & $9.3 E+5$ & $6.6 \mathrm{E}+5$ & $1.8 \mathrm{E}+6$ & $8.7 \mathrm{E}+5$ & $6.3 E+5$ & $2.7 E+3$ & $1.6 \mathrm{E}+4$ & $3.9 \mathrm{E}+4$ & $1.6 \mathrm{E}+4$ & $1.3 \mathrm{E}+4$ & 0.01 & 0.03 & 0.04 & 0.03 & 0.03 \\
\hline ros. $20 \mathrm{sin}$. & $3.6 \mathrm{E}+6$ & $1.0 \mathrm{E}+7$ & $2.5 \mathrm{E}+7$ & $9.8 \mathrm{E}+6$ & $9.2 \mathrm{E}+6$ & $6.0 \mathrm{E}+4$ & $9.4 \mathrm{E}+5$ & $2.3 \mathrm{E}+6$ & $6.4 \mathrm{E}+5$ & $7.6 \mathrm{E}+5$ & 0.02 & 0.08 & 0.12 & 0.09 & 0.09 \\
\hline ros. $50 \mathrm{sin}$. & $2.3 E+8$ & $6.4 \mathrm{E}+8$ & $2.0 \mathrm{E}+9$ & $8.0 \mathrm{E}+8$ & $6.5 \mathrm{E}+8$ & $6.6 \mathrm{E}+7$ & $2.2 \mathrm{E}+8$ & $8.7 \mathrm{E}+8$ & $2.8 \mathrm{E}+8$ & $2.2 \mathrm{E}+8$ & 0.10 & 0.21 & 0.36 & 0.28 & 0.26 \\
\hline ros. $100 \mathrm{sin}$. & $2.5 \mathrm{E}+9$ & $5.2 \mathrm{E}+9$ & $1.1 \mathrm{E}+10$ & $5.2 \mathrm{E}+9$ & $5.0 \mathrm{E}+9$ & $1.5 \mathrm{E}+9$ & $3.2 \mathrm{E}+9$ & $7.2 \mathrm{E}+9$ & $3.2 \mathrm{E}+9$ & $3.2 \mathrm{E}+9$ & .14 & .47 & .56 & .51 & 0.51 \\
\hline 2 lin. & $2.1 \mathrm{E}+0$ & $1.2 \mathrm{E}+0$ & $E+0$ & 1.1E+0 & $1.2 \mathrm{E}+0$ & E-3 & $5.8 \mathrm{E}-3$ & 3.0E-2 & $5.8 \mathrm{E}-3$ & $6.3 \mathrm{E}-3$ & .01 & 1.02 & 07 & .07 & 0.04 \\
\hline $5 \mathrm{lin}$. & $2.2 \mathrm{E}+1$ & $2.0 \mathrm{E}+1$ & 2.6E+1 & $2.2 \mathrm{E}+1$ & $2.0 \mathrm{E}+1$ & $6.9 \mathrm{E}+0$ & $7.3 \mathrm{E}+0$ & $1.1 \mathrm{E}+1$ & $8.8 \mathrm{E}+0$ & $7.2 \mathrm{E}+0$ & 0.05 & 0.17 & 25 & 0.30 & 0.26 \\
\hline rast. 10 lin. & $1.3 \mathrm{E}+2$ & $9.5 \mathrm{E}+1$ & $1.2 \mathrm{E}+2$ & $9.9 \mathrm{E}+1$ & $9.5 \mathrm{E}+1$ & $8.1 \mathrm{E}+1$ & $5.7 \mathrm{E}+1$ & $6.4 \mathrm{E}+1$ & $5.7 \mathrm{E}+1$ & $5.6 \mathrm{E}+1$ & 0.08 & 0.33 & 33 & .39 & 0.41 \\
\hline rast. 20 lin. & $3.9 \mathrm{E}+2$ & $3.6 \mathrm{E}+2$ & $5.0 \mathrm{E}+2$ & $3.7 \mathrm{E}+2$ & $3.6 \mathrm{E}+2$ & $2.5 \mathrm{E}+2$ & $2.2 \mathrm{E}+2$ & $2.9 \mathrm{E}+2$ & $2.2 \mathrm{E}+2$ & $2.2 \mathrm{E}+2$ & 0.10 & 0.39 & 0.41 & .43 & 0.48 \\
\hline rast. 50 lin. & $4.9 \mathrm{E}+3$ & $2.7 \mathrm{E}+3$ & $6.4 \mathrm{E}+3$ & $3.0 \mathrm{E}+3$ & $2.6 \mathrm{E}+3$ & $4.1 \mathrm{E}+3$ & $1.8 \mathrm{E}+3$ & $4.6 \mathrm{E}+3$ & $2.0 \mathrm{E}+3$ & $1.8 \mathrm{E}+3$ & 0.15 & 0.39 & 0.59 & 0.55 & 0.57 \\
\hline rast. 100 lin. & $5.2 E+4$ & 3.0E+4 & $5.1 \mathrm{E}+4$ & 3.0E+4 & $3.0 \mathrm{E}+4$ & 4.7E+4 & $2.5 \mathrm{E}+4$ & 4. $3 E+4$ & $2.4 \mathrm{E}+4$ & $2.5 \mathrm{E}+4$ & 0.17 & 0.40 & 0.66 & 0.72 & 0.74 \\
\hline $2 \sin$ & $1.7 \mathrm{E}+1$ & $2.4 \mathrm{E}+0$ & $2.7 \mathrm{E}+0$ & $2.7 \mathrm{E}+0$ & $2.4 \mathrm{E}+0$ & $9.4 \mathrm{E}+0$ & $9.0 \mathrm{E}-2$ & $2.0 \mathrm{E}-1$ & $1.2 \mathrm{E}-1$ & $8.9 \mathrm{E}-2$ & 0.04 & 0.04 & 0.11 & 0.10 & 0.09 \\
\hline $5 \sin$. & $4.6 \mathrm{E}+1$ & $2.8 \mathrm{E}+1$ & $4.0 \mathrm{E}+1$ & $3.3 E+1$ & $2.8 \mathrm{E}+1$ & $2.5 \mathrm{E}+1$ & $1.1 \mathrm{E}+1$ & $1.6 \mathrm{E}+1$ & 1.3E+1 & $1.1 \mathrm{E}+1$ & 0.06 & 0.25 & 0.27 & 0.27 & 0.29 \\
\hline rast. $10 \mathrm{sin}$. & $3.1 \mathrm{E}+2$ & $2.0 \mathrm{E}+2$ & $2.8 \mathrm{E}+2$ & $2.2 \mathrm{E}+2$ & $2.0 \mathrm{E}+2$ & $1.6 \mathrm{E}+2$ & $7.9 \mathrm{E}+1$ & $1.0 \mathrm{E}+2$ & $8.0 \mathrm{E}+1$ & $7.9 \mathrm{E}+1$ & 0.12 & 0.18 & 0.20 & 0.18 & 0.20 \\
\hline rast. $20 \mathrm{sin}$. & $8.0 \mathrm{E}+2$ & $6.8 \mathrm{E}+2$ & $1.0 \mathrm{E}+3$ & $7.2 \mathrm{E}+2$ & $6.8 \mathrm{E}+2$ & $4.8 \mathrm{E}+2$ & $3.1 \mathrm{E}+2$ & $4.4 \mathrm{E}+2$ & $3.1 \mathrm{E}+2$ & $3.1 \mathrm{E}+2$ & 0.10 & 0.28 & .33 & .29 & .30 \\
\hline rast. $50 \mathrm{sin}$. & $6.7 E+3$ & $8.8 \mathrm{E}+3$ & $1.5 \mathrm{E}+4$ & $9.9 \mathrm{E}+3$ & $8.8 \mathrm{E}+3$ & $4.0 \mathrm{E}+3$ & $5.1 \mathrm{E}+3$ & $9.4 \mathrm{E}+3$ & $5.8 \mathrm{E}+3$ & $5.1 \mathrm{E}+3$ & 0.14 & 0.43 & 0.56 & .50 & 0.48 \\
\hline rast. $100 \mathrm{sin}$. & $2.9 \mathrm{E}+4$ & $3.9 \mathrm{E}+4$ & $5.2 \mathrm{E}+4$ & $3.9 \mathrm{E}+4$ & $3.9 \mathrm{E}+4$ & $2.2 \mathrm{E}+4$ & $3.0 \mathrm{E}+4$ & $4.2 \mathrm{E}+4$ & $3.0 \mathrm{E}+4$ & $3.0 \mathrm{E}+4$ & 0.23 & 0.63 & 0.74 & 0.69 & .70 \\
\hline Best frequen. & 13 & 7 & 1 & 6 & 9 & 22 & 2 & 0 & 6 & 이 & 35 & 1 & 0 & 0 & \\
\hline
\end{tabular}


Table 5: Results for dynPSO on MPB-Noisy benchmark

\begin{tabular}{|c|c|c|c|c|c|c|c|c|c|c|}
\hline \multirow{2}{*}{\multicolumn{2}{|c|}{\begin{tabular}{|l|} 
Metric \\
Benchmark \\
\end{tabular}}} & \multicolumn{3}{|c|}{ BOG } & \multicolumn{3}{|c|}{ BEBC } & \multicolumn{3}{|c|}{ RCS } \\
\hline & & 1.05 & 1.49 & 2.00 & 1.05 & 1.49 & 2.00 & 1.05 & 1.49 & 2.00 \\
\hline noisy (0) & 2 & -22.64 & -142.65 & -155.18 & 153.76 & 33.75 & 21.40 & 0.91 & 0.35 & 0.41 \\
\hline noisy (0) & 20 & -31.62 & -22.31 & -15.99 & 149.78 & 176.51 & 190.97 & 0.84 & 0.90 & 0.94 \\
\hline noisy (0) & 50 & -0.06 & -0.40 & -0.26 & 194.24 & 193.73 & 193.96 & 1.00 & 1.00 & 1.00 \\
\hline noisy (0) & 100 & 0.00 & -0.02 & -0.01 & 187.29 & 187.27 & 187.28 & 1.00 & 1.00 & 1.00 \\
\hline noisy (0.1) & 2 & -22.66 & -23.53 & -144.48 & 153.76 & 153.76 & 32.10 & 0.91 & 0.98 & 0.52 \\
\hline noisy (0.1) & 20 & -32.30 & -22.67 & -16.09 & 149.16 & 176.79 & 190.48 & 0.83 & 0.90 & 0.93 \\
\hline noisy (0.1) & 50 & -0.15 & -0.17 & -0.27 & 194.13 & 194.06 & 193.94 & 1.00 & 1.00 & 1.00 \\
\hline noisy (0.1) & 100 & 0.00 & -0.01 & -0.01 & 187.29 & 187.28 & 187.28 & 1.00 & 1.00 & 1.00 \\
\hline noisy (1) & 2 & -21.59 & -104.53 & -171.54 & 153.76 & 71.47 & 4.05 & 0.91 & 0.60 & 0.14 \\
\hline noisy (1) & 20 & -26.31 & -21.90 & -11.29 & 159.74 & 175.45 & 200.28 & 0.86 & 0.90 & 0.95 \\
\hline noisy (1) & 50 & -0.04 & -0.36 & -0.22 & 194.27 & 193.78 & 194.01 & 1.00 & 1.00 & 1.00 \\
\hline noisy (1) & 100 & 0.00 & -0.01 & -0.01 & 187.29 & 187.28 & 187.28 & 1.00 & 1.00 & 1.00 \\
\hline noisy (10) & & -123.79 & -139.73 & -124.78 & 28.27 & 28.11 & 40.25 & 0.20 & 0.20 & 0.31 \\
\hline noisy (10) & 20 & -0.61 & -0.58 & -0.28 & 223.50 & 223.82 & 224.66 & 1.00 & 1.00 & 1.00 \\
\hline noisy (10) & 50 & 0.00 & -0.02 & -0.01 & 194.31 & 194.29 & 194.30 & 1.00 & 1.00 & 1.00 \\
\hline noisy (10) & 100 & 0.00 & 0.00 & 0.00 & 187.29 & 187.29 & 187.29 & 1.00 & 1.00 & 1.00 \\
\hline Best freque & ency & 4 & 6 & 6 & 4 & 6 & & 5 & 6 & \\
\hline
\end{tabular}

Table 6: Results for pred2p on MPB-Noisy benchmark

\begin{tabular}{|c|c|c|c|c|c|c|c|c|c|c|}
\hline \multirow{2}{*}{\multicolumn{2}{|c|}{\begin{tabular}{|l|} 
Metric \\
Benchmark \\
\end{tabular}}} & \multicolumn{3}{|c|}{ BOG } & \multicolumn{3}{|c|}{ BEBC } & \multicolumn{3}{|c|}{ RCS } \\
\hline & & 1.05 & 1.49 & 2.00 & 1.05 & 1.49 & 2.00 & 1.05 & 1.49 & 2.00 \\
\hline noisy (0) & 2 & -22.70 & -43.93 & -159.17 & 153.76 & 133.19 & 17.14 & 0.91 & 0.93 & 0.34 \\
\hline noisy (0) & 20 & -32.82 & -21.89 & -15.80 & 149.43 & 177.90 & 191.41 & 0.83 & 0.90 & 0.94 \\
\hline noisy (0) & 50 & -0.64 & -0.27 & -0.27 & 193.36 & 193.92 & 193.94 & 1.00 & 1.00 & 1.00 \\
\hline noisy (0) & 100 & -0.07 & -0.04 & -0.01 & 187.21 & 187.25 & 187.28 & 1.00 & 1.00 & 1.00 \\
\hline noisy (0.1) & & -22.67 & -44.50 & -156.18 & 153.76 & 132.65 & 20.00 & 0.91 & 0.87 & 0.37 \\
\hline noisy (0.1) & 20 & -34.03 & -22.65 & -15.53 & 146.15 & 176.18 & 192.06 & 0.83 & 0.90 & 0.94 \\
\hline noisy (0.1) & 50 & -0.26 & -0.24 & -0.27 & 193.90 & 193.96 & 193.93 & 1.00 & 1.00 & 1.00 \\
\hline noisy (0.1) & 100 & -0.09 & -0.03 & -0.01 & 187.18 & 187.26 & 187.28 & 1.00 & 1.00 & 1.00 \\
\hline noisy (1) & 2 & -21.76 & -79.04 & -167.25 & 153.76 & 97.49 & 8.41 & 0.91 & 0.73 & 0.19 \\
\hline noisy (1) & 20 & -29.27 & -21.66 & -11.15 & 151.31 & 176.18 & 200.69 & 0.85 & 0.90 & 0.95 \\
\hline noisy (1) & 50 & -0.14 & -0.35 & -0.23 & 194.11 & 193.79 & 194.00 & 1.00 & 1.00 & 1.00 \\
\hline noisy (1) & 100 & -0.05 & -0.03 & -0.01 & 187.23 & 187.26 & 187.28 & 1.00 & 1.00 & 1.00 \\
\hline noisy (10) & 2 & -123.95 & -134.22 & -129.14 & 28.75 & 33.34 & 33.48 & 0.20 & 0.24 & 0.28 \\
\hline noisy (10) & 20 & -0.93 & -0.58 & -0.29 & 222.27 & 223.84 & 224.64 & 0.99 & 1.00 & 1.00 \\
\hline noisy (10) & 50 & -0.01 & -0.02 & -0.01 & 194.30 & 194.29 & 194.30 & 1.00 & 1.00 & 1.00 \\
\hline noisy (10) & 100 & 0.00 & 0.00 & 0.00 & 187.29 & 187.29 & 187.29 & 1.00 & 1.00 & 1.00 \\
\hline \multicolumn{2}{|c|}{ Best frequency } & 9 & 3 & 4 & 11 & 2 & 3 & 11 & 2 & 3 \\
\hline
\end{tabular}


Table 7: Results for pred3 on MPB-Noisy benchmark

\begin{tabular}{|lr|rrr|rrr|rrr|}
\hline \multicolumn{1}{|c|}{} & \multicolumn{3}{|c|}{ BOG } & \multicolumn{3}{c|}{ BEBC } & \multicolumn{3}{c|}{ RCS } \\
\hline \multicolumn{2}{|l|}{ Benchmark } & 1.05 & 1.49 & 2.00 & 1.05 & 1.49 & 2.00 & 1.05 & 1.49 & 2.00 \\
\hline noisy (0) & 2 & -23.11 & -104.97 & $-\mathbf{1 6 0 . 7 5}$ & 153.76 & 71.79 & $\mathbf{1 5 . 3 2}$ & 0.91 & 0.58 & $\mathbf{0 . 3 1}$ \\
noisy (0) & 20 & -17.07 & $\mathbf{- 2 4 . 1 8}$ & -16.15 & 191.60 & $\mathbf{1 7 5 . 4 6}$ & 191.06 & 0.91 & $\mathbf{0 . 9 0}$ & 0.93 \\
noisy (0) & 50 & $\mathbf{- 1 . 4 1}$ & -0.32 & -0.28 & $\mathbf{1 9 1 . 9 1}$ & 193.82 & 193.92 & $\mathbf{0 . 9 9}$ & 1.00 & 1.00 \\
noisy (0) & 100 & $-\mathbf{0 . 1 7}$ & -0.11 & -0.03 & $\mathbf{1 8 7 . 0 8}$ & 187.15 & 187.26 & $\mathbf{1 . 0 0}$ & 1.00 & 1.00 \\
\hline noisy (0.1) & 2 & -23.05 & -99.15 & $\mathbf{- 1 5 3 . 6 8}$ & 153.76 & 77.57 & $\mathbf{2 2 . 2 9}$ & 0.91 & 0.55 & $\mathbf{0 . 4 1}$ \\
noisy (0.1) & 20 & -15.80 & $\mathbf{- 2 4 . 6 7}$ & -16.30 & 192.33 & $\mathbf{1 7 4 . 8 2}$ & 190.40 & 0.91 & $\mathbf{0 . 8 9}$ & 0.93 \\
noisy (0.1) & 50 & $-\mathbf{0 . 7 5}$ & -0.31 & -0.28 & $\mathbf{1 9 3 . 0 3}$ & 193.83 & 193.92 & $\mathbf{1 . 0 0}$ & 1.00 & 1.00 \\
noisy (0.1) & 100 & $-\mathbf{0 . 1 2}$ & -0.12 & -0.02 & $\mathbf{1 8 7 . 1 3}$ & 187.14 & 187.26 & $\mathbf{1 . 0 0}$ & 1.00 & 1.00 \\
\hline noisy (1) & 2 & -22.42 & -111.65 & $\mathbf{- 1 6 6 . 9 8}$ & 153.76 & 64.59 & $\mathbf{7 . 6 9}$ & 0.91 & 0.52 & $\mathbf{0 . 1 8}$ \\
noisy (1) & 20 & $\mathbf{- 3 3 . 2 1}$ & -21.90 & -11.33 & $\mathbf{1 4 4 . 2 8}$ & 175.34 & 199.96 & $\mathbf{0 . 8 3}$ & 0.90 & 0.95 \\
noisy (1) & 50 & $-\mathbf{0 . 2 9}$ & -0.22 & -0.22 & $\mathbf{1 9 3 . 8 0}$ & 193.97 & 193.99 & $\mathbf{1 . 0 0}$ & 1.00 & 1.00 \\
noisy (1) & 100 & $-\mathbf{0 . 1 6}$ & -0.10 & -0.02 & $\mathbf{1 8 7 . 0 8}$ & 187.16 & 187.27 & $\mathbf{1 . 0 0}$ & 1.00 & 1.00 \\
\hline noisy (10) & 2 & $\mathbf{- 1 2 6 . 3 4}$ & -124.87 & -118.78 & $\mathbf{3 1 . 5 6}$ & 41.15 & 39.48 & $\mathbf{0 . 2 0}$ & 0.29 & 0.33 \\
noisy (10) & 20 & $\mathbf{- 1 . 1 1}$ & -0.53 & -0.28 & $\mathbf{2 2 1 . 5 8}$ & 223.90 & 224.64 & $\mathbf{0 . 9 9}$ & 1.00 & 1.00 \\
noisy (10) & 50 & -0.01 & $-\mathbf{0 . 0 2}$ & -0.01 & 194.29 & $\mathbf{1 9 4 . 2 9}$ & 194.30 & $\mathbf{1 . 0 0}$ & 1.00 & 1.00 \\
noisy (10) & 100 & $-\mathbf{0 . 0 1}$ & 0.00 & 0.00 & $\mathbf{1 8 7 . 2 8}$ & 187.29 & 187.29 & $\mathbf{1 . 0 0}$ & 1.00 & 1.00 \\
\hline Best frequency & 10 & 3 & 3 & 10 & 3 & 3 & 11 & 2 & 3 \\
\hline
\end{tabular}

Table 8: Results for pred3p on MPB-Noisy benchmark

\begin{tabular}{|lr|rrr|rrr|rrr|}
\hline Metric & \multicolumn{3}{|c|}{ BOG } & \multicolumn{3}{|c|}{ BEBC } & \multicolumn{3}{|c|}{ RCS } \\
\hline \multicolumn{2}{|l|}{ Benchmark } & 1.05 & 1.49 & 2.00 & 1.05 & 1.49 & 2.00 & 1.05 & 1.49 & 2.00 \\
\hline noisy (0) & 2 & -23.09 & -100.37 & $-\mathbf{1 5 5 . 6 5}$ & 153.76 & 76.36 & $\mathbf{2 0 . 4 4}$ & 0.91 & 0.62 & $\mathbf{0 . 3 8}$ \\
noisy (0) & 20 & -16.92 & $\mathbf{- 2 4 . 5 9}$ & -16.92 & 191.73 & $\mathbf{1 7 4 . 5 6}$ & 189.46 & 0.91 & $\mathbf{0 . 8 9}$ & 0.93 \\
noisy (0) & 50 & $\mathbf{- 1 . 4 0}$ & -0.31 & -0.28 & $\mathbf{1 9 1 . 9 3}$ & 193.83 & 193.93 & $\mathbf{0 . 9 9}$ & 1.00 & 1.00 \\
noisy (0) & 100 & $-\mathbf{0 . 1 8}$ & -0.11 & -0.02 & $\mathbf{1 8 7 . 0 6}$ & 187.15 & 187.26 & $\mathbf{1 . 0 0}$ & 1.00 & 1.00 \\
\hline noisy (0.1) & 2 & -23.04 & -109.83 & $\mathbf{- 1 5 4 . 4 2}$ & 153.76 & 66.87 & $\mathbf{2 1 . 4 6}$ & 0.91 & 0.49 & $\mathbf{0 . 3 9}$ \\
noisy (0.1) & 20 & -15.93 & $\mathbf{- 2 5 . 9 6}$ & -15.16 & 192.03 & $\mathbf{1 7 1 . 8 9}$ & 192.80 & 0.91 & $\mathbf{0 . 8 9}$ & 0.94 \\
noisy (0.1) & 50 & $-\mathbf{0 . 7 5}$ & -0.32 & -0.27 & $\mathbf{1 9 3 . 0 3}$ & 193.82 & 193.93 & $\mathbf{1 . 0 0}$ & 1.00 & 1.00 \\
noisy (0.1) & 100 & $-\mathbf{0 . 1 5}$ & -0.11 & -0.02 & $\mathbf{1 8 7 . 1 0}$ & 187.16 & 187.27 & $\mathbf{1 . 0 0}$ & 1.00 & 1.00 \\
\hline noisy (1) & 2 & -22.44 & -119.38 & $\mathbf{- 1 6 4 . 7 9}$ & 153.76 & 56.87 & $\mathbf{9 . 7 2}$ & 0.91 & 0.49 & $\mathbf{0 . 2 1}$ \\
noisy (1) & 20 & $\mathbf{- 3 2 . 6 2}$ & -21.98 & -11.35 & $\mathbf{1 4 5 . 6 6}$ & 175.32 & 199.76 & $\mathbf{0 . 8 4}$ & 0.90 & 0.95 \\
noisy (1) & 50 & $-\mathbf{0 . 3 0}$ & -0.23 & -0.22 & $\mathbf{1 9 3 . 7 9}$ & 193.96 & 194.00 & $\mathbf{1 . 0 0}$ & 1.00 & 1.00 \\
noisy (1) & 100 & $-\mathbf{0 . 1 5}$ & -0.11 & -0.02 & $\mathbf{1 8 7 . 1 0}$ & 187.16 & 187.27 & $\mathbf{1 . 0 0}$ & 1.00 & 1.00 \\
\hline noisy (10) & 2 & $\mathbf{- 1 2 6 . 9 7}$ & -120.45 & -121.70 & $\mathbf{3 0 . 6 8}$ & 46.24 & 36.86 & $\mathbf{0 . 2 0}$ & 0.32 & 0.31 \\
noisy (10) & 20 & $\mathbf{- 1 . 1 3}$ & -0.53 & -0.27 & $\mathbf{2 2 1 . 5 6}$ & 223.90 & 224.66 & $\mathbf{0 . 9 9}$ & 1.00 & 1.00 \\
noisy (10) & 50 & -0.01 & $-\mathbf{0 . 0 2}$ & -0.01 & 194.29 & $\mathbf{1 9 4 . 2 9}$ & 194.30 & $\mathbf{1 . 0 0}$ & 1.00 & 1.00 \\
noisy (10) & 100 & $-\mathbf{0 . 0 1}$ & 0.00 & 0.00 & $\mathbf{1 8 7 . 2 8}$ & 187.29 & 187.29 & $\mathbf{1 . 0 0}$ & 1.00 & 1.00 \\
\hline Best frequency & 10 & 3 & 3 & 10 & 3 & 3 & 11 & 2 & 3 \\
\hline
\end{tabular}

